\title{
Chromosome aberrations involving 10q22: report of three overlapping interstitial deletions and a balanced translocation disrupting C10orf11
}

\author{
Andreas Tzschach ${ }^{\star 1}$, Anne-Marie Bisgaard ${ }^{2}$, Maria Kirchhoff ${ }^{2}$, Luitgard M Graul-Neumann ${ }^{3,4}$, \\ Heidemarie Neitzel ${ }^{4}$, Stephanie Page ${ }^{5}$, Alischo Ahmed ${ }^{1}$, Ines Müller ${ }^{1}$, Fikret Erdogan ${ }^{1}$, Hans-Hilger Ropers ${ }^{1}$, \\ Vera M Kalscheuer ${ }^{1}$ and Reinhard Ullmann ${ }^{1}$
}

Interstitial deletions of chromosome band 10q22 are rare. We report on the characterization of three overlapping de novo 10q22 deletions by high-resolution array comparative genomic hybridization in three unrelated patients. Patient 1 had a $7.9 \mathrm{Mb}$ deletion in 10q21.3-q22.2 and suffered from severe feeding problems, facial dysmorphisms and profound mental retardation. Patients 2 and 3 had nearly identical deletions of 3.2 and $3.6 \mathrm{Mb}$, the proximal breakpoints of which were located at an identical low-copy repeat. Both patients were mentally retarded; patient 3 also suffered from growth retardation and hypotonia. We also report on the results of breakpoint analysis by array painting in a mentally retarded patient with a balanced chromosome translocation 46,XY,t(10;13)(q22;p13)dn. The breakpoint in 10q22 was found to disrupt C10orf11, a brain-expressed gene in the common deleted interval of patients 1-3. This finding suggests that haploinsufficiency of C10orf11 contributes to the cognitive defects in 10q22 deletion patients.

European Journal of Human Genetics (2010) 18, 291-295; doi:10.1038/ejhg.2009.163; published online 21 October 2009

Keywords: deletion 10q22; array CGH; C10orf11; mental retardation; balanced chromosome translocation

\section{INTRODUCTION}

Interstitial deletions of chromosome band 10q22 are rare. ${ }^{1-3}$ Patients with 10q22 deletions suffer from mental retardation but lack characteristic additional clinical features that would allow the delineation of a recognizable '10q22 deletion syndrome'. So far, little is known about the contribution of individual genes within the deleted intervals to cognitive defects. Strategies to elucidate the role of individual genes for specific clinical problems are the analysis of deletions of different sizes and positions within the region of interest, mutation screening of candidate genes in patients with matching phenotypes or the analysis of breakpoints of balanced chromosome rearrangements.

In this study, we report on the characterization of overlapping interstitial 10q22 deletions by high-resolution array comparative genomic hybridization (CGH) in three unrelated mentally retarded patients. We also report on a mentally retarded patient with a balanced chromosome translocation in whom the analysis of the 10q22 breakpoint revealed the disruption of C10orf11.

\section{MATERIALS AND METHODS}

\section{Array CGH}

Whole-genome analysis using an Agilent $244 \mathrm{~K}$ array. Whole-genome array CGH analysis was performed using 244 or $400 \mathrm{~K}$ oligonucleotide arrays (Agilent, Santa Clara, CA, USA) according to protocols provided by the manufacturer. Image analysis, normalization and annotation were based on Feature Extraction 9.1 (Agilent) using default settings, and visualization of data was performed with CGHPRO software. $^{4}$

Customized array. Fine mapping of chromosomal breakpoints in patients 1 and 2 was carried out using a customized oligonucleotide array covering the region chr10:74 119676-79455990 (HG18; NCBI 36.1 ) with a resolution of approximately $200-300 \mathrm{bp}$. Array design was performed by means of eArray (Agilent). Repetitive sequences and segmental duplications were not included. Arrays were printed by Agilent and hybridized according to the manufacturer's recommendations for array CGH experiments.

\section{Array painting}

Flow-sorting of metaphase chromosomes was performed as described previously. 5,6 Both derivative chromosomes were amplified using the GenomiPhi V2 DNA Amplification kit (GE Healthcare, Piscataway, NJ, USA) following the manufacturer's protocol. One microgram of each amplification product was labeled with $\mathrm{Cy} 3$ and $\mathrm{Cy} 5$, using Agilent's Genomic DNA Enzymatic Labeling Kit Plus (Agilent). To each labeling reaction $100 \mathrm{ng}$ of genomic control DNA was added to ensure proper placement of the grid after image analysis. Hybridization was performed on a $244 \mathrm{~K}$ whole-genomic DNA array using the array CGH protocol as described above.

${ }^{1}$ Department Human Molecular Genetics, Max Planck Institute for Molecular Genetics, Berlin, Germany; ${ }^{2}$ Department of Clinical Genetics, Rigshospitalet, Copenhagen, Denmark; ${ }^{3}$ Institute of Medical Genetics, Charité-Universitätsmedizin Berlin, Berlin, Germany; ${ }^{4}$ Institute of Human Genetics, Charité-Universitätsmedizin Berlin, Berlin, Germany; ${ }^{5}$ Westfall Pediatrics, Rochester, NY, USA

${ }^{*}$ Correspondence: Dr A Tzschach, Department Human Molecular Genetics, Max Planck Institute for Molecular Genetics, Ihnestr 73, 14195 Berlin, Germany. Tel: +49 308413 1416; Fax: +49 308413 1383; E-mail: tzschach@molgen.mpg.de

Received 22 April 2009; revised 11 August 2009; accepted 28 August 2009; published online 21 October 2009 


\section{C10orf11 sequence analysis}

The six exons and exon-intron boundaries of C10orf11 were PCRamplified using genomic DNA of patient 4. Specific products were sequenced by Sanger sequencing. Oligonucleotide primers are available on request.

\section{CLINICAL REPORTS}

\section{Patient 1}

The girl was born after an uneventful pregnancy in the 39th gestational week with low birth weight ( $2775 \mathrm{~g}$, below third centile) but normal length $(49 \mathrm{~cm}, 3 \mathrm{rd}-10$ th centile) and head circumference $(34 \mathrm{~cm}, 50$ th centile). After birth, she had respiratory problems (which necessitated surveillance in an intensive care unit for 2 weeks) and hypotonia. Dysmorphic features noted after birth included hypertelorism, low-set ears, retrognathia, small nose and elongated, finger-like thumbs (Figure 1a). She had severe feeding problems, which prompted the placement of a gastric feeding tube at the age of 3 months. She also suffered from gastroesophageal reflux and underwent surgery for a Nissen fundoplication at the age of 15 months. Psychomotor development was severely retarded. On examination at the age of 3 years and 9 months, she was found to be unable to speak and could not walk independently. She still could not eat by mouth. Hearing tests revealed profound sensorineural hearing loss in her right ear and moderate hearing loss in the left ear. Height $(92 \mathrm{~cm})$ and weight $(10.5 \mathrm{~kg})$ were below third centile; head circumference was normal $(49 \mathrm{~cm}, 25-50$ th centile). A brain MRI scan at the age of 16 months demonstrated delayed myelination. An EEG was inconspicuous. A muscle biopsy failed to show any structural abnormalities. Chromosome analysis (GTG banding) revealed an interstitial deletion in one homolog of chromosome 10 (46,XX, $\operatorname{del}(10)(\mathrm{q} 22.1 \mathrm{q} 22.3) \mathrm{dn})$. The karyotypes of the parents were normal. The patient has two healthy older sisters.

\section{Patient 2}

The patient was born after an unremarkable pregnancy to healthy and nonconsanguineous parents (birth measurements: weight, $3406 \mathrm{~g}$ (50th centile); length, $53 \mathrm{~cm}$ ( $>75$ th centile); OFC, $37 \mathrm{~cm}$ (>90th centile)). Psychomotor development was retarded. On examination at the age of 4 years and 6 months, he only spoke a few words and communicated using about 15 signs. Hearing, vision and body measurements were normal (height, $111 \mathrm{~cm}$ (25th centile); weight, $19 \mathrm{~kg}$ (50th centile); OFC, $53.5 \mathrm{~cm}$ (90th centile)). He was a happy and charming boy. Facial dysmorphic signs included hypertelorism ( $>2$ SD), low-set ears, small mouth, retrognathia and brachycephaly (cephalic index was 94) (Figures 1b and c). He had clinodactyly of the fifth fingers (Figure 1d). CT and MR brain scans revealed no brain abnormalities. Investigation by array CGH using a BAC array ${ }^{7}$ revealed an interstitial deletion of approximately $3.2 \mathrm{Mb}$ in $10 \mathrm{q} 22$.

\section{Patient 3}

The clinical details of this patient and the characterization of her 10q22 deletion by FISH analysis have been reported earlier. ${ }^{3}$ The main abnormalities of the girl comprised mental retardation, short stature, hypertelorism and strabismus. Comparison of the FISH results of this patient and the BAC array CGH results of patient 2 suggested a nearly identical size and position of both 10q22 deletions.

\section{Patient 4}

The boy was born in the 35th gestational week by cesarean section after a twin pregnancy with normal birth measurements (weight, $2420 \mathrm{~g}$ (25th centile); length, $48 \mathrm{~cm}$ (50th centile)).

On examination at the age of 4 years, all body measurements were in the upper normal range (height, $109 \mathrm{~cm}$ (90th centile); weight, $19.5 \mathrm{~kg}$ (90-97th centile); head circumference, $53 \mathrm{~cm}$ (75-90th centile)). Psychomotor development was retarded. At the age of 4 years,
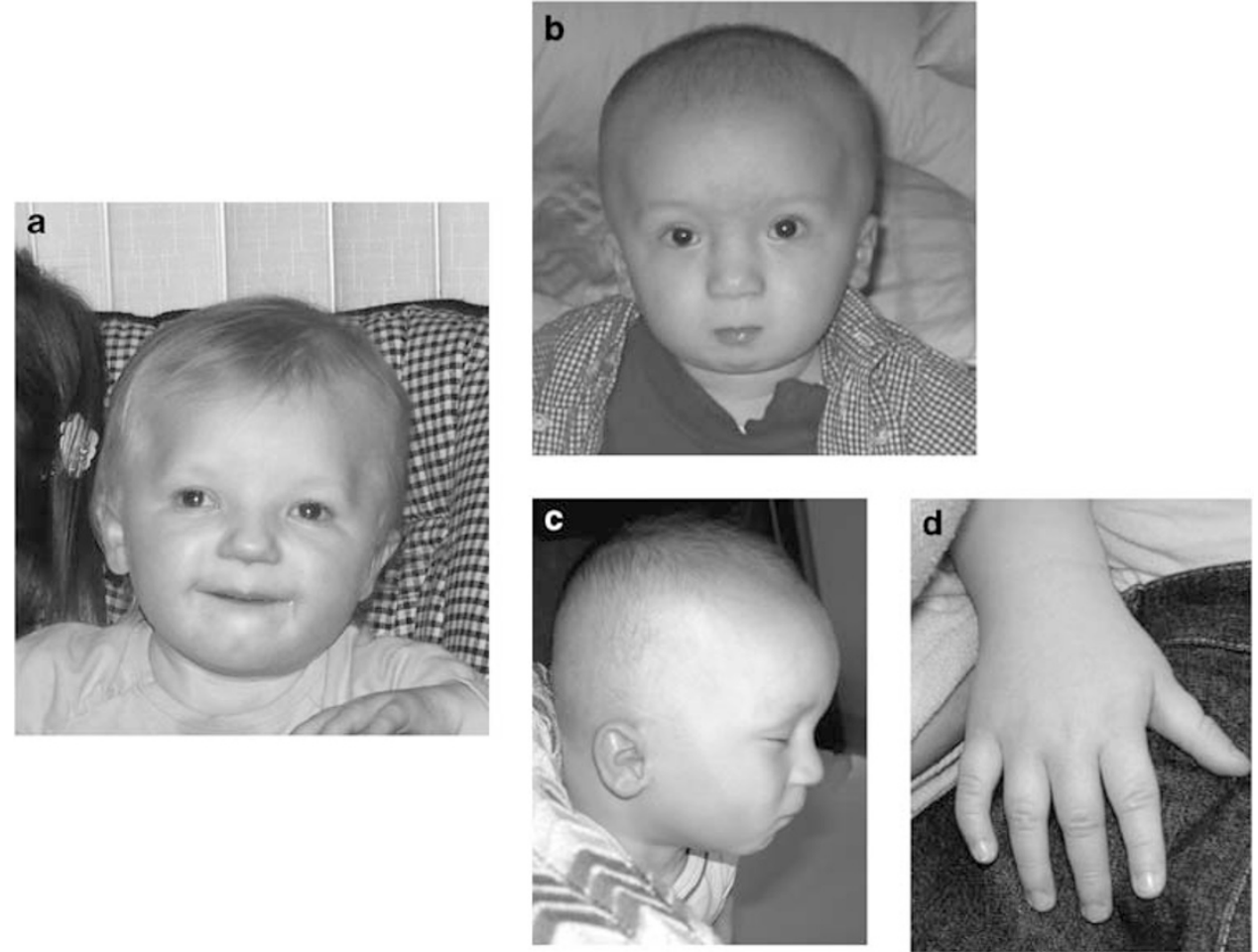

Figure 1 (a) Facial features of patient 1 at the age of 2 years. Note hypertelorism and low-set ears. (b-d) Facial features and right hand of patient 2 at the age of 13 months. Note small mouth, retrognathia, low-set ears, brachycephaly and clinodactyly of the fifth finger. 
Table 1 Summary of clinical features of patients $1-4$ and of the patient reported by Cook et al ${ }^{1}$

\begin{tabular}{|c|c|c|c|c|c|}
\hline Clinical features & Patient 1 & Patient 2 & Patient $3^{3}$ & Patient 4 & Cook et al ${ }^{1}$ \\
\hline Karyotype & $\begin{array}{l}\text { 46,XX,del(10) } \\
(q 21.3 q 22.2)\end{array}$ & $\begin{array}{l}\text { 46,XY,del(10) } \\
(q 22.2 q 22.3)\end{array}$ & $\begin{array}{l}46, X X, \operatorname{del}(10) \\
(q 22.2 q 22.3)\end{array}$ & $\begin{array}{l}46, \mathrm{XY}, \mathrm{t}(10 ; 13) \\
(\mathrm{q} 22.3 ; \mathrm{p} 13) \mathrm{dn}\end{array}$ & $\begin{array}{l}46, X Y, \operatorname{del}(10) \\
\text { (q22.1q22.3) }\end{array}$ \\
\hline Size of deletion & $7.9 \mathrm{Mb}$ & $3.2 \mathrm{Mb}$ & $3.6 \mathrm{Mb}$ & Balanced translocation & Not determined \\
\hline Age of examination & 3 years 9 months & 4 years 6 months & 2 years 6 months & 5 years & 8 months \\
\hline Sex & Female & Male & Female & Male & Male \\
\hline Developmental delay & + & + & + & + & + \\
\hline Growth deficiency & + & - & + & - & + \\
\hline OFC & 25-50th centile & 75-90th centile & 10th centile & 75-90th centile & 25th centile \\
\hline Muscular hypotonia & + & - & + & - & 'poor head control' \\
\hline Strabismus & - & - & + & + & + \\
\hline Ocular hypertelorism & + & + & + & - & + \\
\hline Posteriorly rotated ears & + & - & - & - & + \\
\hline Retrognathia & + & + & - & - & + \\
\hline Fifth finger clinodactyly & - & + & - & - & + \\
\hline Dental lamina cysts & + & - & - & - & + \\
\hline Genital abnormalities & - & - & Small labia minora & - & $\begin{array}{c}\text { Bilateral } \\
\text { undescended testes }\end{array}$ \\
\hline Other & $\begin{array}{c}\text { Unilateral deafness, } \\
\text { gastroesophageal } \\
\text { reflux, long and lean thumbs }\end{array}$ & - & - & Myopia & - \\
\hline
\end{tabular}

he only spoke a few single words. He had started walking at the age of 12 months, but had an ataxic gait. He had strabismus and mild myopia, but neither facial dysmorphic signs nor other malformations. Brain MRI, EEG and fundoscopy investigations revealed normal results. A routine chromosome analysis detected a de novo balanced translocation between the long arm of one homolog of chromosome 10 and the short arm of one homolog of chromosome 13: 46,XY,t (10;13)(q22.3;p13)dn. Array CGH analysis using a $400 \mathrm{~K}$ oligonucleotide array (Agilent) detected no potentially disease-associated copy number variations. Both parents and his healthy nonidentical twin brother had normal karyotypes.

The clinical features of patients 1-4 have been summarized in Table 1.

\section{RESULTS}

All chromosome coordinates given below are according to UCSC Genome Browser HG18 (NCBI build 36.1).

\section{Whole-genome array CGH (Agilent 244K)}

Patient 1. Whole-genome array CGH analysis using a $244 \mathrm{~K}$ Agilent oligo array demonstrated that the 10q22 deletion of patient 1 had a proximal border at genomic position chr10:69329 292; and the distal breakpoint was at chr10:77 267180 (Figure 2a). Thus, the deletion has a size of $7.9 \mathrm{Mb}$ (Figure 3).

\section{Customized array analysis}

Patients 2 and 3. To characterize the similar 10q22 deletions of patients 2 and 3 in more detail, we analyzed both patients by a customized high-density oligo array for this region. As a result, we could demonstrate that the deletion of patient 2 is approximately $400 \mathrm{~kb}$ smaller than the deletion of patient 3 (Figures $2 \mathrm{~b}, \mathrm{c}$ and 3 ). In patient 2, the proximal breakpoint is at chr10:75 197738 and the distal breakpoint is at chr10:78 463676. Thus, the size of the deletion is $3.2 \mathrm{Mb}$.

In patient 3, the proximal breakpoint is at chr10:75049389 and the distal breakpoint is at chr10:78 711382 . The size of the deletion is $3.6 \mathrm{Mb}$.
Breakpoint analysis/array painting of the $t(10 ; 13)$ translocation Patient 4. Hybridization of the flow-sorted translocation chromosomes $\operatorname{der}(10)$ and $\operatorname{der}(13)$ on an Agilent $244 \mathrm{~K}$ chip (array painting) localized the breakpoint in $10 \mathrm{q}$ between genomic positions chr10:77625748 and chr10:77 632595 (Figure 4). Thus, the breakpoint is located within intron 4 of the C10orf11 (CDA017) gene, and the translocation resulted in the disruption of this six-exon gene (Figure 3).

The breakpoint in the short arm of chromosome 13 was not analyzed because $13 \mathrm{p}$ contains no known protein coding genes (only rRNA genes).

C10orf11 sequence analysis

Resequencing of the six exons and exon-intron boundaries of C10orf11 revealed no aberrations from the reference sequence.

\section{DISCUSSION}

Patient 1, who carried the largest 10q22 deletion (7.9 Mb), had more complex clinical features (including feeding problems, facial dysmorphisms and severe mental retardation) compared with patients 2 and 3 who had smaller deletions (Table 1; Figure 3 ). The clinical problems of patients 2 and 3 were similar but not identical. Only patient 3 had retarded growth and hypotonia, which may have been a consequence of her slightly larger deletion $(3.6 \mathrm{Mb}$ compared with $3.2 \mathrm{Mb}$ in patient 2) (Figure 3). The facial features in the presently known patients with 10q22 deletions are rather unspecific and do not allow the delineation of a clinically recognizable '10q22 deletion syndrome'.

The deletions of patients 2 and 3 had nearly identical proximal breakpoints, which were located close to a known low-copy repeat (LCR) (Figure 3). Although the absence of LCRs at the distal breakpoints of these deletions makes nonallelic homologous recombination as a cause of these aberrations rather unlikely, the common LCR at the proximal boundary may still constitute a predisposing factor for their formation. ${ }^{8,9}$ The presence of LCRs at only one breakpoint has already been observed in numerous other interstitial 
a

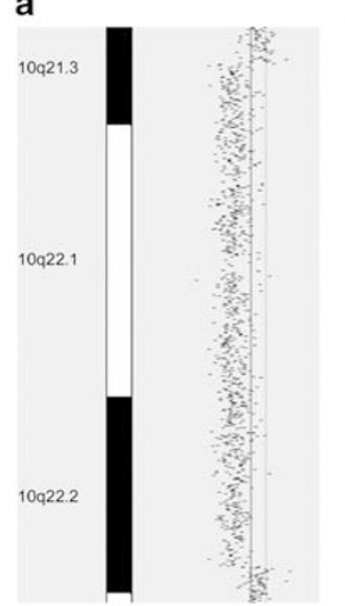

b

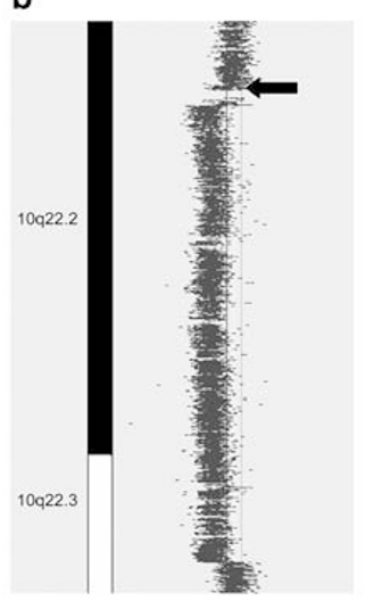

C

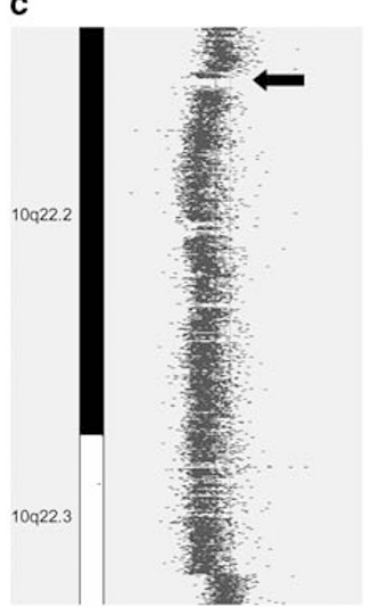

d

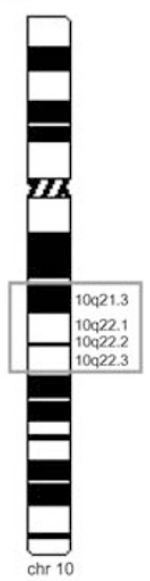

Figure 2 (a) Partial result of the whole-genome array CGH (244K) analysis in patient 1 depicting the $7.9 \mathrm{Mb}$ deletion in 10q21.3-q22.2. (b and c) Results of the customized array CGH analyses of patients 2 (b) and 3 (c). The arrows mark the gap in the array due to a low-copy repeat, which also marks the proximal breakpoint of both deletions. (d) Ideogram of chromosome 10 highlighting the region of the deletions (red box).

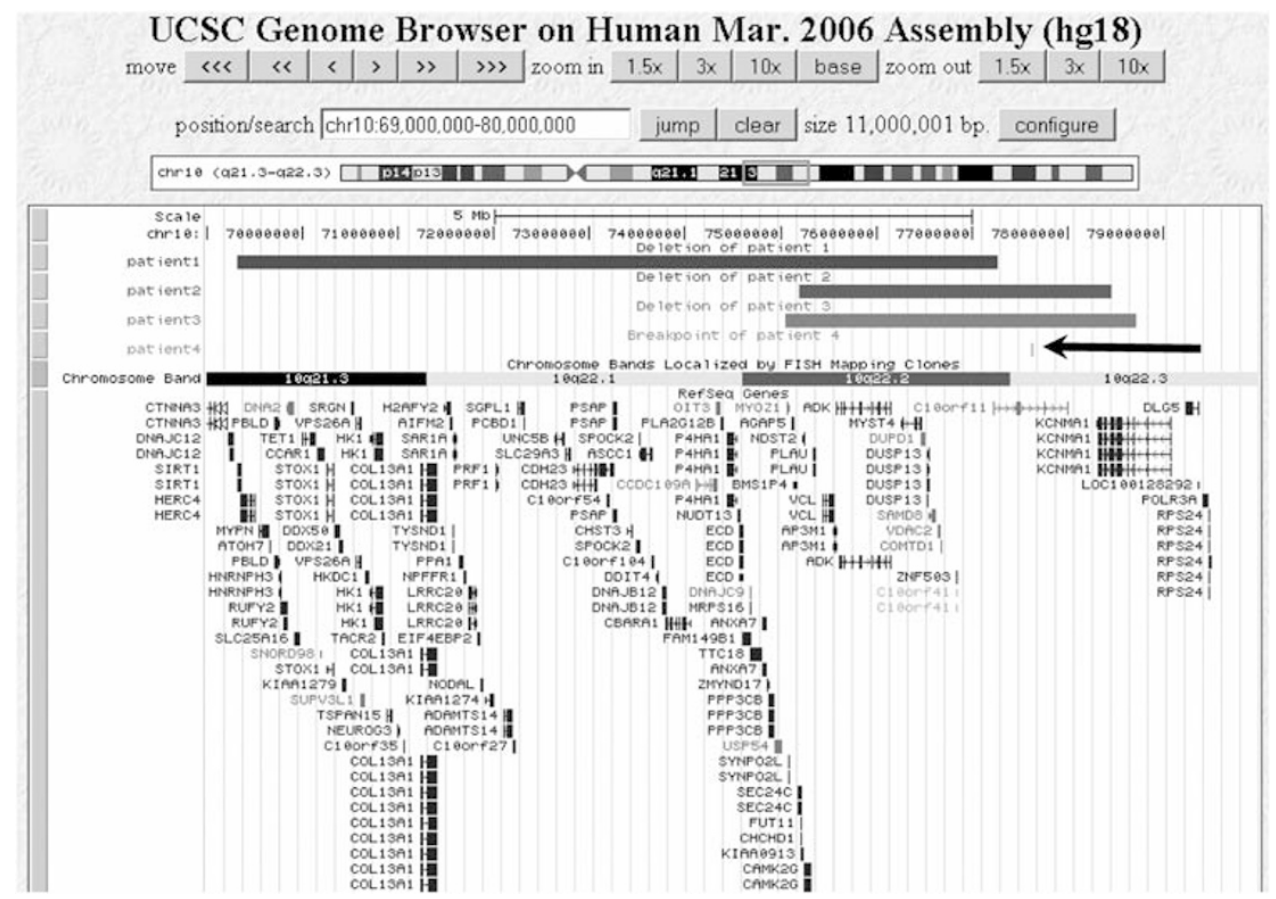

Figure 3 Screenshot from the UCSC Genome Browser showing the positions of the deletions of patient 1 (blue bar), patient 2 (brown bar), patient 3 (green bar) and the position of the chromosome breakpoint of patient 4 (red tick mark and arrow). Note that the translocation breakpoint of patient 4 is located in intron 4 of C10orf11.

deletion syndromes, but the mechanisms leading to the deletions in such cases remain elusive. ${ }^{7,10-12}$

Developmental delay and mental retardation, which are the prominent clinical problems in all $\operatorname{del}(10)$ (q22) patients, are most likely caused by haploinsufficiency of genes within the deletions, or by position effects on neighboring genes. None of the 23 genes (MYOZ1, SYNPO2L, AGAP5, SEC24C, FUT11, CHCHD1, KIAA0913, NDST2, CAMK2G, PLAU, VCL, AP3M1, ADK, MYST4, DUPD1, DUPD13, SAMD8, VDAC2, COMTD1, C10orf41, ZNF503, C10orf11, KCNMA1) within the common deleted interval of patients 2 and 3 has been associated with mental retardation so far.

The disruption of one of the genes in the interval - C10orf11 - by a chromosome breakpoint of a balanced translocation in a mentally retarded boy (patient 4 in this study) suggests that haploinsufficiency of this brain-expressed gene may be sufficient to cause mental retardation. However, this assumption needs to be confirmed by the identification of additional C10orf11 mutations in other patients, and by elucidating the biological role of its little-characterized gene 
a

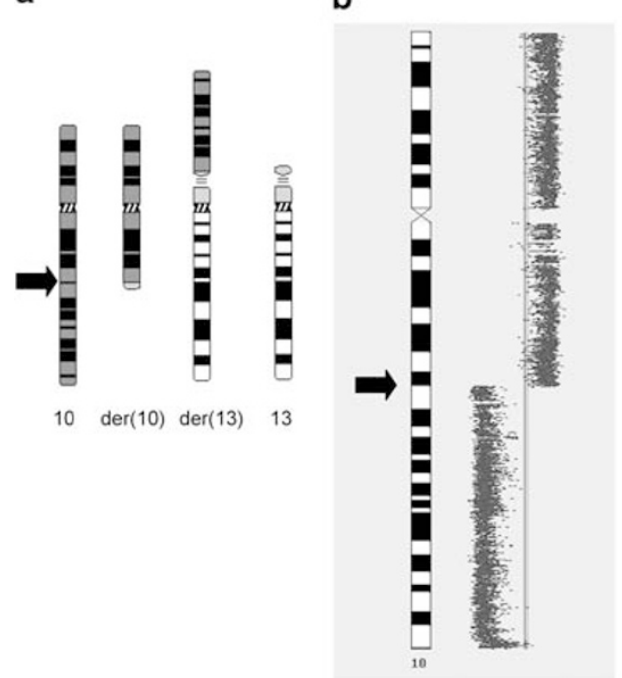

C

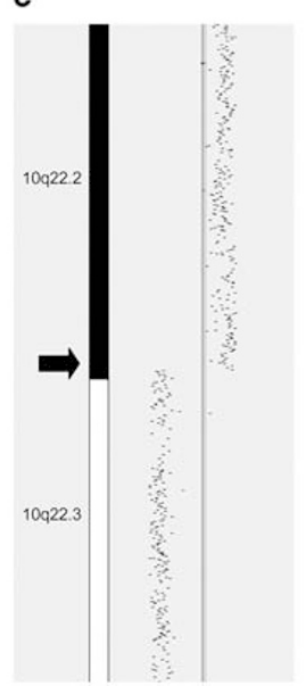

d

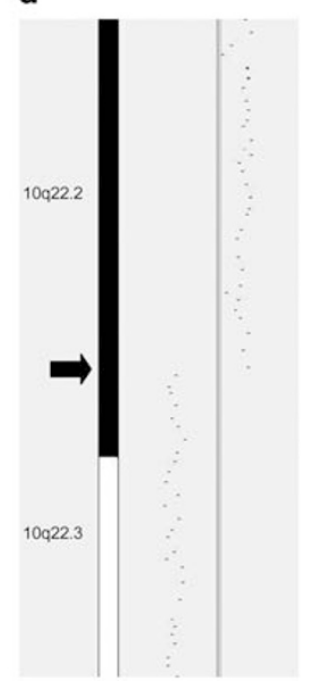

Figure 4 (a) Partial ideogram of translocation 46,XY,t(10;13)(q22.3;p13)dn of patient 4 depicting the derivative chromosomes 10 (red) and 13 (white) and their normal homologs. (b) Result of the array painting of the flow-sorted derivative chromosomes on a $244 \mathrm{~K}$ oligo array CGH chip (Agilent). (c and d) Enlarged depiction of the breakpoint region in chromosome 10q22. The breakpoint is marked by arrows.

product. Position effects of the chromosome breakpoint on neighboring genes may also contribute to clinical problems.

Mutations in the vinculin gene, VCL, which is also harbored by the common deleted interval, have been associated with dilated cardiomyopathy in adult patients. ${ }^{13}$ Patients with $10 \mathrm{q} 22$ deletions comprising VCL should therefore undergo regular cardiac examinations.

In summary, this report broadens our knowledge of the clinical consequences of 10q22 deletions. Analysis of a chromosome breakpoint suggests that haploinsufficiency of C10orf11 might contribute to mental retardation and has thus illustrated the potential of balanced chromosome rearrangements to elucidate major causative genes in microdeletion syndromes.

\section{ACKNOWLEDGEMENTS}

We thank the parents of patients for their support, as well as Hannelore Madle, Susanne Freier, Nadine Nowak and Corinna Menzel for expert technical assistance. Array CGH was supported by the German Nationales Genomforschungsnetzwerk (Grant 01GR0203) and by the Max Planck Innovation Fund. This article is dedicated to the memory of Dr Ines Krause-Plonka.

1 Cook L, Weaver DD, Hartsfield Jr JK, Vance GH: De novo 10q22 interstitial deletion. J Med Genet 1999; 36: 71-72.
2 Balciuniene J, Feng N, lyadurai $\mathrm{K}$ et al: Recurrent 10q22-q23 deletions: a genomic disorder on 10q associated with cognitive and behavioral abnormalities. Am J Hum Genet 2007; 80: 938-947.

3 Tzschach A, Krause-Plonka I, Menzel C et al: Molecular cytogenetic analysis of a de novo interstitial chromosome 10q22 deletion. Am J Med Genet A 2006; 140: 1108-1110.

4 Chen W, Erdogan F, Ropers HH, Lenzner S, Ullmann R: CGHPRO - a comprehensive data analysis tool for array CGH. BMC Bioinformatics 2005; 6: 85.

5 Arkesteijn G, Jumelet E, Hagenbeek A, Smit E, Slater R, Martens A: Reverse chromosome painting for the identification of marker chromosomes and complex translocations in leukemia. Cytometry 1999; 35: 117-124.

6 Chen W, Kalscheuer V, Tzschach A et al: Mapping translocation breakpoints by nextgeneration sequencing. Genome Res 2008; 18: 1143-1149.

7 Erdogan F, Chen W, Kirchhoff M et al: Impact of low copy repeats on the generation of balanced and unbalanced chromosomal aberrations in mental retardation. Cytogenet Genome Res 2006; 115: 247-253.

8 Lupski JR, Stankiewicz P: Genomic disorders: molecular mechanisms for rearrangements and conveyed phenotypes. PLoS Genet 2005; 1: e49.

9 Shaw CJ, Lupski JR: Implications of human genome architecture for rearrangementbased disorders: the genomic basis of disease. Hum Mol Genet 2004; 13 (Spec No 1): R57-R64.

10 Emanuel BS: Molecular mechanisms and diagnosis of chromosome 22q11.2 rearrangements. Dev Disabil Res Rev 2008; 14: 11-18.

11 Schubert C: The genomic basis of the Williams-Beuren syndrome. Cell Mol Life Sci 2008; 66: 1178-1197.

12 Urban AE, Korbel JO, Selzer R et al: High-resolution mapping of DNA copy alterations in human chromosome 22 using high-density tiling oligonucleotide arrays. Proc Natl Acad Sci USA 2006; 103: 4534-4539.

13 Olson TM, Illenberger S, Kishimoto NY, Huttelmaier S, Keating MT, Jockusch BM: Metavinculin mutations alter actin interaction in dilated cardiomyopathy. Circulation 2002; 105: 431-437. 